\title{
A Lower Bound for Computing Oja Depth
}

\author{
Greg Aloupis* Erin McLeish*
}

September 17, 2004

\begin{abstract}
Let $S=\left\{s_{1}, \ldots, s_{n}\right\}$ be a set of points in the plane. The Oja depth of a query point $\theta$ with respect to $S$ is the sum of the areas of all triangles $\left(\theta, s_{i}, s_{j}\right)$. This depth may be computed in $O(n \log n)$ time in the RAM model of computation. We show that a matching lower bound holds in the algebraic decision tree model. This bound also applies to the computation of the Oja gradient, the Oja sign test, and to the problem of computing the sum of pairwise distances among points on a line.
\end{abstract}

Keywords: algorithms, computational geometry, computational statistics, data depth, bivariate medians

*School of Computer Science, McGill University. \{athens,mcleish\}@cgm.cs.mcgill.ca 


\section{Introduction}

The depth of a point $\theta$ with respect to a data set $S=\left\{s_{1}, \ldots, s_{n}\right\}$ in the plane is a quantitative measure of how central $\theta$ is in $S$. Several notions of depth exist, and typically a multivariate median definition can be formed by taking a point with minimum/maximum depth. Such notions are of great interest to the statistical community. We refer the reader to $[1,2]$ for recent results with a similar flavor to what is presented here, and for further introductory references to the topic of statistical depth. The main result in [1] involves lower bounds on the computation of halfspace depth [8] and simplicial depth [5]. For both depths, the given bound of $\Omega(n \log n)$ was in the algebraic decision tree model and matched known upper bounds in the RAM model. The same upper bound also applies to the computation of the Oja depth [6] of a point $\theta$ [7]. This depth is defined to be the sum of the areas of all triangles $\left(\theta, s_{i}, s_{j}\right)$, as shown in Figure 1.

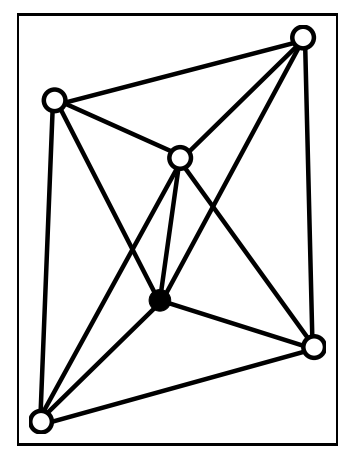

Figure 1: The Oja depth of the black point with respect to the set of white points is the sum of the areas of all triangles that include the black point as a vertex. 
Here we show that the computation of Oja depth takes $\Omega(n \log n)$ time. This bound also holds for the computation of the Oja gradient, the Oja sign test, and for the problem of computing the sum of pairwise distances among points on a line.

\section{The Lower Bound}

We reduce the problem of Set Equality to the problem of computing Oja depth. It is known that determining whether two sets of real numbers are equal requires $\Omega(n \log n)$ time in the algebraic decision tree model [3]. We show that by performing some work that takes $O(n)$ time and then making a few Oja depth queries, we can answer the question of Set Equality. This implies that computing Oja depth takes $\Omega(n \log n)$ time.

Suppose that we are given two sets of real numbers, $A=\left\{a_{1}, \ldots, a_{n}\right\}$ and $B=\left\{b_{1}, \ldots, b_{n}\right\}$. We wish to know if these two sets are equal. Here, we describe a procedure that answers this question. First we assign planar coordinates to the elements of our given sets, in linear time. Every element $a_{i}$ simply becomes the point $\left(a_{i}, 0\right)$, and the same holds for all $b_{i}$. Then we select a query point $\theta$ at $(0,2)$. The area of any triangle formed by $\theta$ and two points taken from $A$ and/or $B$ is equal to the distance between those two points. Thus, in our construction, the Oja depth of $\theta$ with respect to some set $S$ is the sum of pairwise distances among elements of $S$. Let $d_{S}$ denote this depth (or sum of distances). 
Theorem 2.1 Computing the Oja depth of a point with respect to a set of $n$ points in the plane requires $\Omega(n \log n)$ time in the algebraic decision tree model.

\section{Proof:}

Suppose that we are given two sets of real numbers $A$ and $B$, each of size $n$. We first construct corresponding planar point sets as described above, and compute the Oja depths $d_{A}, d_{B}$ and $d_{A \cup B}$. The claim is that sets $A$ and $B$ are equal if and only if $2\left(d_{A}+d_{B}\right)=d_{A \cup B}$. This immediately implies that computing Oja depth must take $\Omega(n \log n)$ time.

Let $C=A \cup B$, and reorder the elements in $C$ so that they are in increasing order: $c_{1} \leq c_{2} \leq \ldots \leq c_{2 n}$. We can rewrite $d_{C}$ as a sum of the length of the intervals $\left|c_{i+1}-c_{i}\right|$ by noticing that the interval length is added to the sum $d_{C}$ exactly $i(2 n-i)$ times. So $d_{C}=\sum_{i=1}^{2 n-1} i(2 n-i)\left|c_{i+1}-c_{i}\right|$. Since $i$ is the number of points in $C$ which are smaller than or equal to $c_{i}$, we can write $i=p_{i}+q_{i}$ where $p_{i}=\left|a \in A, a \leq c_{i}\right|$ and $q_{i}=\left|b \in B, b \leq c_{i}\right|$. Thus $d_{C}=\sum_{i=1}^{2 n-1}\left(p_{i}+q_{i}\right)\left(2 n-p_{i}-q_{i}\right)\left|c_{i+1}-c_{i}\right|$. Similarly, we can use these intervals to rewrite the sums $d_{A}$ and $d_{B}$. In the sum $d_{A}$, the value $\left|c_{i+1}-c_{i}\right|$ will be added exactly $p_{i}\left(n-p_{i}\right)$ times. So $d_{A}=\sum_{i=1}^{2 n-1} p_{i}\left(n-p_{i}\right)\left|c_{i+1}-c_{i}\right|$ and $d_{B}=\sum_{i=1}^{2 n-1} q_{i}\left(n-q_{i}\right)\left|c_{i+1}-c_{i}\right|$. Now we are ready to compare $2\left(d_{A}+d_{B}\right)$ to $d_{C}$. From the above expressions it is easy to check that

$$
d_{C}-2\left(d_{A}+d_{B}\right)=\sum_{i=1}^{2 n-1}\left|c_{i+1}-c_{i}\right|\left(p_{i}-q_{i}\right)^{2}
$$


Now assume that the sets $A$ and $B$ are equal. Then clearly $p_{i}=q_{i}$ for all $i$, and thus $2\left(d_{A}+d_{B}\right)=d_{C}$. On the other hand, assume the sets $A$ and $B$ differ on at least one point. Then there exists some $i$ for which $p_{i} \neq q_{i}$. We can see this by selecting the first $i$ in order for which $a_{i} \neq b_{i}$. For this $i$ we will have that $p_{i} \neq q_{i}$. Therefore, if $A \neq B$ we have that

$$
d_{C}-2\left(d_{A}+d_{B}\right)=\sum_{i=1}^{2 n-1}\left|c_{i+1}-c_{i}\right|\left(p_{i}-q_{i}\right)^{2}>0
$$

Thus, using a few simple steps that take $O(n)$ time, and computing the Oja depths for sets $A, B$ and $A \cup B$ we can answer the question of Set Equality. This implies that the computation of Oja depth requires $\Omega(n \log n)$ time.

\section{Remarks}

Given two points in the plane, define a vector that has magnitude equal to the distance between the two points, and that has direction orthogonal to the line through the points and pointed away from the origin. Given a set $S$ and a query point $\theta$, taken to be the origin for simplicity, the Oja gradient at $\theta$ is the sum of all vectors defined by pairs of points in $S$ (see Figure 2). This gradient may be computed in $O(n \log n)$ time [7]. It is used for the computation of the Oja median, which is the point in the plane that has 
highest Oja depth (see [2]).

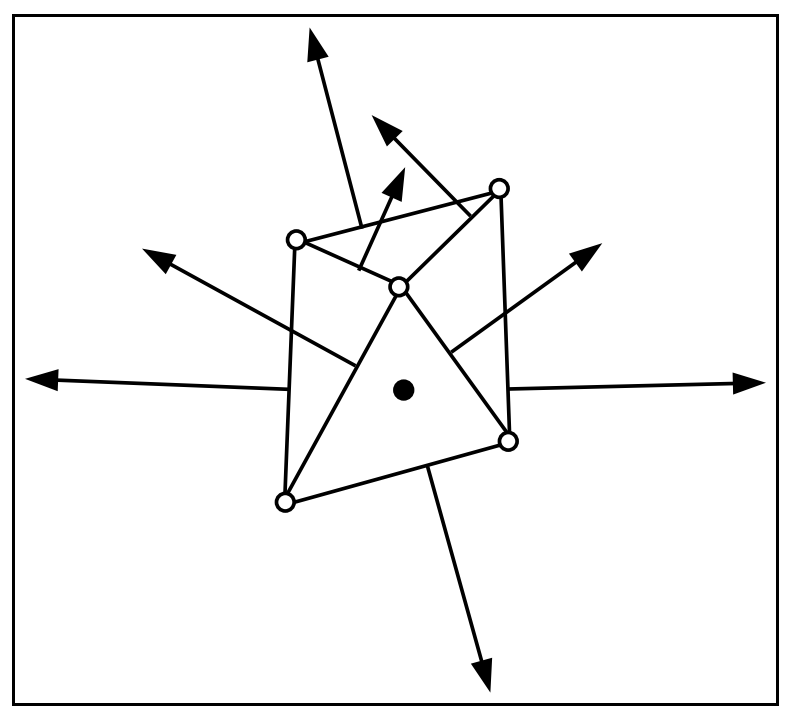

Figure 2: The Oja gradient of the black vertex with respect to the set of white vertices is a sum of vectors, each of which is orthogonal to a segment between two white vertices. Not all segments and vectors are illustrated.

Suppose that we are given a planar point set that is contained on a line $\ell$. The Oja gradient at any point at unit distance from $\ell$ will have the same value as the Oja depth of a point that is two units of distance from $\ell$. This implies that computing the Oja gradient takes $\Omega(n \log n)$ time.

Finally, Brown and Hettmansperger show that the Oja gradient can be used as a bivariate sign test [4]. Thus our lower bound holds in this case too. 


\section{References}

[1] G. Aloupis, C. Cortes, F. Gomez, M. Soss, and G. Toussaint. Lower bounds for computing statistical depth. Computational Statistics and Data Analysis, 40:223-229, 2002.

[2] G. Aloupis, S. Langerman, M. Soss, and G. Toussaint. Algorithms for bivariate medians and a Fermat-Torricelli problem for lines. Computational Geometry, 26, 2003.

[3] M. Ben-Or. Lower bounds for algebraic computation trees. In Proc. 15th Ann. ACM Sympos. Theory Comput., pages 80-86, 1983.

[4] B.M. Brown and T.P. Hettmansperger. An afine invariant bivariate version of the sign test. J.R. Statist. Soc. B, 51(1):117-125, 1989.

[5] R. Liu. On a notion of data depth based upon random simplices. The Annals of Statistics, 18:405-414, 1990.

[6] H. Oja. Descriptive statistics for multivariate distributions. Statistics and Probability Letters, 1:327-332, 1983.

[7] P. Rousseeuw and I. Ruts. Bivariate location depth. Applied Statistics, 45:516-526, 1996.

[8] J. Tukey. Mathematics and the picturing of data. In Proceedings of the International Congress of Mathematicians, pages 523-531, Vancouver, 1975. 